\title{
Examination of the Ovarian Reserve after Generation of Unilateral Rudimentary Uterine Horns in Rats
}

\author{
Hasan Toyganözü, ${ }^{1}$ Hakan Nazik, ${ }^{1}$ Raziye Narin, ${ }^{1}$ Deniz Satar, ${ }^{1}$ Mehmet Ali Narin, \\ Sinem Büyüiknacar, ${ }^{2}$ Murat Api, ${ }^{3}$ and Hakan Aytan ${ }^{4}$ \\ ${ }^{1}$ Department of Obstetrics and Gynecology, Adana Numune Education and Research Hospital, 01330 Adana, Turkey \\ ${ }^{2}$ Department of Pharmacology, Cukurova University Medical School, Adana, Turkey \\ ${ }^{3}$ Department of Obstetrics and Gynecology, Zeynep Kamil Education and Research Hospital, Istanbul, Turkey \\ ${ }^{4}$ Department of Obstetrics and Gynecology, Mersin University Medical School, Mersin, Turkey
}

Correspondence should be addressed to Hakan Nazik; drhakannazik@gmail.com

Received 18 November 2013; Accepted 30 December 2013; Published 6 February 2014

Academic Editors: R. Abir and T. Levy

Copyright (C) 2014 Hasan Toyganözü et al. This is an open access article distributed under the Creative Commons Attribution License, which permits unrestricted use, distribution, and reproduction in any medium, provided the original work is properly cited.

Objective. The purpose of this experimental rat model study is to evaluate the changes in the ovarian environment after excision of the rudimentary horn. Methods. Ten female Wistar albino rats were used in this study. One $\mathrm{cm}$ of right uterine horn length was excised in the first operation. Two months after the first operation, all animals were sacrificed to obtain ovaries for histological examination. Mann-Whitney $U$ test and Student's $t$-test were used for statistical analysis purposes. Statistical significance was defined as $P<0.005$. Results. The number of primordial follicles $(P=0.415)$, primary follicles $(P=0.959)$, preantral follicles $(P=0.645)$, antral follicles $(P=0.328)$, and Graafian follicles $(P=0.721)$ was decreased and the number of atretic follicles $(P=0.374)$ increased in the right ovarian side. Howeve,r this difference was not found to be statistically significant. Conclusion. The results of this experimental rat model study suggest that the excision of rudimentary horn could have negative effects on ipsilateral ovarian functions.

\section{Introduction}

Congenital Müllerian anomalies account for the majority of uterus anomalies. The prevalence of uterine anomaly in the general population is estimated to be $0.5 \%$ [1]. Unicornuate uterus occurs in the event of arrest or failure of development of one Müllerian duct. The incidence of unicornuate uterus in uterine anomalies has been reported at 14\% [2]. The classification of the American Society for Reproductive Medicine (ASRM) divides this anomaly group into four subgroups: cavity rudimentary horn communicating with the unicornuate uterus, noncommunicating cavity rudimentary horn, no-cavity rudimentary horn, and unicornuate uterus without rudimentary horn. A unicornuate uterus may lead to many gynecological or obstetric complications. They include infertility, dysmenorrhea, endometriosis, and premature birth [3, 4]. If pregnancy occurs in the rudimentary horn, then there is high risk of uterine rupture. So, it is suggested that the rudimentary horn is excised as soon as it is diagnosed due to such risks [4]. No sufficient data is available covering the effects of the excision of a rudimentary uterine horn that is on the ovarian reserve located on the same side. It has been previously shown that tubal ligation and hysterectomy affected the blood flow to the ovary and decreased the ovarian reserve [5-13].

Besides this, the diminished reserve may be due to some other factors such that uterus may have a mechanical supporting role to the ovary and removal may negatively affect this function which may consequently cause decrease in ovarian reserve. Another theory may be that there may be some unknown factors for blood supply or survival being transferred by contact with the uterus and removal may prevent this transfer. From here, we can say that theoretically 
the excision of rudimentary horn will likely negatively affect the ovarian reserve on the same side. The purpose of this experimental rat model study is to evaluate the changes in the ovarian environment after excision of the rudimentary horn.

\section{Material and Method}

This experimental study was conducted after obtaining the Ethical Committee Consent of the Experimental Medicine Research Institute, Faculty of Medicine, Çukurova University. The surgical arm of this study was conducted at the Animal Laboratory of the Department of Pharmacology, Çukurova University. And the histopathological morphometric analyses were made at the Pathology Laboratory of Adana Numune Education and Research Hospital. This study employed 10 female Wistar Albino rats weighing 180-220 grams. Adult female Wistar albino rats were housed in individual metabolic cages for two weeks to acclimatize them to the study surroundings.

All rats were anesthetized with ketamine hydrochloride (60 mg/kg IP) and xylazine hydrochloride (10 mg/kg IP). The abdominal skin was shaved and antisepsis was performed with a $10 \%$ povidone-iodine solution before the surgery. Using a sterile technique, a $3 \mathrm{~cm}$ vertical midline incision was performed and both uterine horns were exposed. About $1 \mathrm{~cm}$ of the right uterine horn was excised distally. The midline incision was closed in two layers by $2 / 0$ polyglactin sutures: fascia and skin. The procedure was performed by two investigators. After the surgery, the rats were separately caged on the first day, and then all were kept in the same cage after making sure that they were healthy. A second laparotomy was performed 8 weeks after initial surgical procedure and the rats were sacrificed with high-dose ( 3 times the normal dose) ketamine anesthesia. Ovaries of the rats were excised. The ovaries were carefully separated from the surrounding fat layer and placed into a $10 \%$ formaldehyde solution for histopathological examination.

Tissues harvested from right and left ovaries for light microscopic examination were fixed in $10 \%$ buffered formalin solution. The materials were placed in cassettes, which were then put in a tissue processor. Paraffinized tissues removed from the tissue processor were then formed into blocks. A routine $4 \mu \mathrm{m}$ section was collected from the paraffin blocks, and one serial section in every $50 \mu \mathrm{m}$ was collected from each block, placed on a slide and stained with Hematoxylin and Eosin (H\&E). H\&E-stained preparations were examined using Olympus (Olympus BX53, Japan) light microscopy. Primordial (PrF), primary (PF), preantral, secondary, Graafian, atretic follicles and corpus luteum in each ovarian tissue were counted. The theca-interstitial cells in the ovarian stroma were evaluated. Their images were taken with an Olympus DP26 digital camera.

SPSS 11.5 (SPSS demo, Chicago, IL) software was used for statistical analysis. Kolmogorov-Smirnov test with Lillefor's correction was used to test whether the data used in the study were normally distributed. Normally distributed data were compared with a $t$-test used in two independent groups, and nonnormally distributed data was compared with the MannWhitney $U$ test. Statistical significance was defined as $P<$ 0.05 .

\section{Results}

One of the 10 rats included in the study was lost due to anesthesia-related complications following the first surgery. One other rat was lost one week later after the operation. An examination showed that the rat died due to an infection. The ovarian follicle counts of the remaining 8 rats in the study and a comparison of the ovarian follicles on the side of the resection (right ovary) and the other ovary (left ovary) are shown in Table 1.

Primordial follicles, corpus lutea, and atretic follicles were compared using $t$-test as they were normally distributed, and the primary follicles, preantral follicles, secondary follicles, and Graafian follicles were evaluated using the MannWhitney $U$ test as they were nonnormally distributed.

The number of primordial follicles $(P=0.415)$, primary follicles $(P=0.959)$, preantral follicles $(P=0.645)$, antral follicles $(P=0.328)$, and Graafian follicles $(P=0.721)$ was decreased and the number of atretic follicles $(P=0.374)$ increased in the right ovarian. However, this difference was not found to be statistically significant.

Images of the histopathological examination of right and left ovaries are given in the Figures 1 and 2. Increased atretic follicles, calcification, and fibrosis are seen in the right ovary. The right ovary also clearly shows reduced primary and antral follicles (Figure 1).

Section of the left ovary is seen in Figure 2. It is observed that all stages of follicular development containing primordial follicles, primary follicle, and antral follicle (AF) are clearly seen in the left ovary sample. The ovarian reserve is better compared with the right ovary (Figure 2).

\section{Discussion}

Women with unicornuate uterus suffer many obstetric and gynecological problems. Women with unicornuate uterus demonstrated higher rates of infertility, endometriosis, hematometra, and dysmenorrhea $[3,4]$. As the probability of rupture and associated intra-abdominal bleeding risk is higher in the cavity rudimentary horn during pregnancy, this horn is recommended to be excised immediately after diagnosis [4].

There is insufficient data about the effects of the excision of the uterus rudimentary horn over the ovarian reserve on the same side. It has been previously demonstrated that tubal ligation and hysterectomy affected blood flow to the ovary and reduced the ovarian reserves [5-13]. From here, theoretically, it is likely that the excision of the rudimentary horn will also negatively affect the reserve of the ovary on the same side. The purpose of this study is to investigate whether the partial excision of the uterine horn affects the reserve of the ipsilateral ovary in a rat model. There is insufficient data covering the effects of the excision of the uterus rudimentary horn over the ovarian reserve on the same side. Previous 
TABLE 1: Comparison of the follicles in the right and left ovaries.

\begin{tabular}{|c|c|c|c|c|c|}
\hline \multirow{2}{*}{ Ovary } & \multicolumn{2}{|c|}{ Right side with resection } & \multicolumn{2}{|c|}{ Left ovary } & \multirow{2}{*}{$P$} \\
\hline & Mean \pm SD & Median (min-max) & Mean \pm SD & Median (min-max) & \\
\hline Primordial follicle & $3.75 \pm 3.41$ & $2.5(0-11)$ & $5.37 \pm 4.27$ & $4.5(1-15)$ & 0.415 \\
\hline Primary follicle & $0.87 \pm 1.12$ & $0.5(0-3)$ & $0.75 \pm 0.7$ & $1(0-2)$ & 0.959 \\
\hline Preantral follicle & $2.5 \pm 1.69$ & $2(0-5)$ & $2.8 \pm 1.72$ & $2.5(0-5)$ & 0.645 \\
\hline Secondary follicle & $0.75 \pm 1.16$ & $0(0-3)$ & $1.25 \pm 0.88$ & $1.5(0-2)$ & 0.328 \\
\hline Graafian follicle & $0.12 \pm 0.35$ & $0(0-1)$ & $0.25 \pm 0.46$ & $0(0-1)$ & 0.721 \\
\hline Corpus luteum & $4.3 \pm 3.5$ & $4(0-10)$ & $6.8 \pm 2.5$ & $6.5(4-11)$ & 0.129 \\
\hline Atretic follicle & $3.0 \pm 2.2$ & $2.5(0-6)$ & $2.12 \pm 1.55$ & $1.5(1-5)$ & 0.374 \\
\hline
\end{tabular}

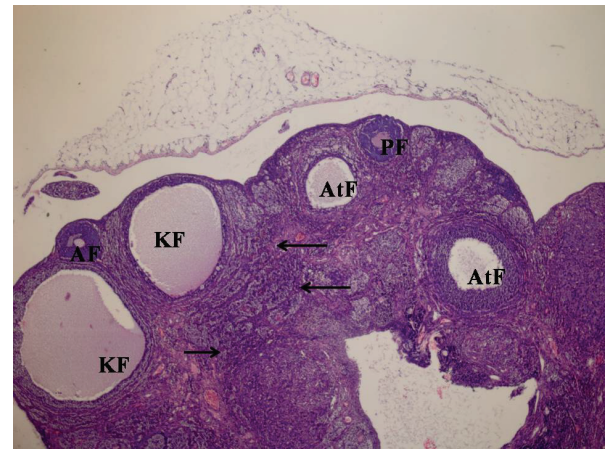

Figure 1: A slight decrease in primary follicles (PF) and antral follicles $(\mathrm{AF})$ in right ovary sample, as well as an increase in theca interna cells in the more intensively observed cystic follicles (KF), atretic follicles (AtF), and ovarian stroma (arrows) $(\mathrm{H} \& \mathrm{E} \times 40)$.

studies investigated the effects of hysterectomy and tubal ligation over the ovarian reserve.

Among studies investigating the ovarian reserve after tubal ligation, elevated gonadotropin levels have been reported in some studies [5-7], whereas no changes have been reported by others [14-17]. Kelekci et al., in their prospective controlled study in 2006, have investigated ovarian reserve after tubal ligation. In this study, the main outcome measurements were blood levels of follicle-stimulating hormone (FSH), luteinizing hormone ( $\mathrm{LH})$, and estradiol $\left(\mathrm{E}_{2}\right)$, ovarian volume, number of antral follicles and Doppler study of ovarian stromal artery pulsatile index (PI), and maximum velocity $\left(\mathrm{V}_{\max }\right)$ on the third of the cycle immediately before and one and twelve months after the surgical intervention. A statistically meaningful increase was found in FSH and PI values after tubal ligation in the first month, but no statistically meaningful difference was found after twelve months. As a result, it was found that tubal ligation caused no differences in the ovarian reserve [18].

There is lack of consensus among studies investigating ovarian functions after hysterectomy. Some studies found that ovarian functions were affected [8-11], while others found the opposite; that is, they were not affected $[19,20]$. Souza et al. investigated ovarian histology and function before and after total abdominal hysterectomy in 25 patients. Immediately before hysterectomy, bilateral ovarian biopsies were performed and, 12 months later, all patients underwent a second

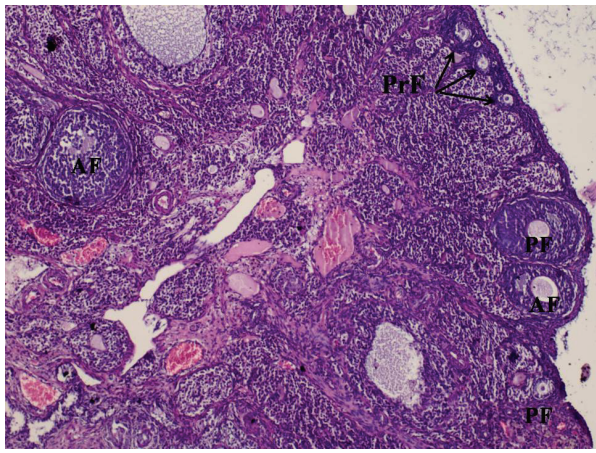

FIGURE 2: Ovarian tissue sample where all stages of follicular development containing primordial follicles (PrF-arrows), primary follicle (PF), and antral follicle (AF) are clearly seen in the left ovary sample $(\mathrm{H} \& \mathrm{E} \times 40)$.

ovarian biopsy through laparoscopy. Histologic study of the ovaries one year after total abdominal hysterectomy showed stromal cell hyperplasia, thickening of the tunica albuginea, and a significant decrease of follicular reserve, follicular cysts, and corpora albicantia. There was no significant difference in the number of atretic follicles and corpora lutea. The serum levels of all hormones studied were unchanged 12 months after the surgical procedures [10].

Özdamar et al. investigated the ovarian morphology and FSH values on Wistar albino rats after hysterectomy. In their histological examination 6 months after hysterectomy, they reported that the ovarian cortex was fully covered with corpora lutea and the number of cystic follicles and atretic follicles increased, and they found only several secondary follicles and tertiary follicles, but no primary follicles. There is also a meaningful increase in FSH blood level [21]. In a similar study, Tapisiz et al. investigated the ovarian morphology and FSH, Inhibin A, and Inhibin B levels after hysterectomy on Wistar albino rats. Histologic examination of the ovaries was performed 100 days after hysterectomy and the findings were found to be similar to those of Özdamar et al. However, no significant change was found in hormonal levels [22].

Anti-Müllerian hormone is an important marker to show the ovarian reserve $[23,24]$. Atabekoğlu et al. investigated the AMH levels after hysterectomy in order to evaluate ovarian reserves in 22 patients who underwent total abdominal 
hysterectomy. A reduction was found in AMH levels after hysterectomy, but it was statistically insignificant [25].

In this study, we created rudimentary horns in Wistar albino rats and then investigated the effects of the excision of rudimentary horn on the ovarian functions on the same side. Reviewing the literature revealed no similar studies. We found that the excision of rudimentary horn affected the particular ovary but this effect was statistically negligible. The possible mechanism might be the damaging of vascular structures passing to the ovary from the uterus due to the procedure and the reduction of blood flow to the ovary secondary to it.

Finding of no statistically meaningful difference between the two ovaries with regarding their reserves in our study might be due to our smaller number of cases. Thus, the study was launched with a total of 10 animals, and the results were based on 8 of them after loss of two animals. The minimum number of animals is recommended in studies on experimental animals. From here, exactly determination of whether or not the difference between the two groups is statistically meaningful can only be achieved through studies containing higher number of cases. So, studies with wider series are needed to cover the subject discussed here.

The most important limitation of this study is that, as in all studies on animals, the findings may not be accurately reflecting the effects on humans. In addition to the species difference between humans and animals, it should be noted that results can differ among members of the same species as well. This can be overcome by using inbred rats, but no inbred rats were used in this study due to inability to supply them.

As a result, we suggest the excision of the particular rudimentary horn in the event of potential complications in certain forms of the rudimentary horn anomaly. However, it is unclear how the ovarian reserve on the same side will be affected from this procedure at the end of this excision. This study shows that the excision procedure will negatively affect the ovarian reserve on the same side on a rat model. To the best of our knowledge, this is the first study that addresses this particular subject matter.

\section{Conflict of Interests}

The authors declare that there is no conflict of interests regarding the publication of this paper.

\section{References}

[1] G. G. Nahum, "Uterine anomalies: how common are they, and what is their distribution among subtypes?" Journal of Reproductive Medicine for the Obstetrician and Gynecologist, vol. 43, no. 10 , pp. 877-887, 1998.

[2] E. Zanetti, L. R. Ferrari, and G. Rossi, "Review article. Classification and radiographic features of uterine malformations: hysterosalpingographic study," British Journal of Radiology, vol. 51, no. 603, pp. 161-170, 1978.

[3] D. Reichman, M. R. Laufer, and B. K. Robinson, "Pregnancy outcomes in unicornuate uteri: a review," Fertility and Sterility, vol. 91, no. 5, pp. 1886-1894, 2009.
[4] P. K. Heinonen, "Unicornuate uterus and rudimentary horn," Fertility and Sterility, vol. 68, no. 2, pp. 224-230, 1997.

[5] A. U. Hakverdi, C. E. Taner, A. C. Erden, and O. Satici, "Changes in ovarian function after tubal sterilization," Advances in Contraception, vol. 10, no. 1, pp. 51-56, 1994.

[6] E. Wu, B. Xiao, W. Yan, H. Li, and B. Wu, "Hormonal profile of the menstrual cycle in Chinese women after tubal sterilization," Contraception, vol. 45, no. 6, pp. 583-593, 1992.

[7] E. Radwanska, S. K. Headley, and P. Dmowski, "Evaluation of ovarian function after tubal sterilization," Journal of Reproductive Medicine for the Obstetrician and Gynecologist, vol. 27, no. 7, pp. 376-384, 1982.

[8] A. Oldenhave, L. J. B. Jaszmann, W. T. A. M. Everaerd, and A. A. Haspels, "Hysterectomized women with ovarian conservation report more severe climacteric complaints than do normal climacteric women of similar age," American Journal of Obstetrics and Gynecology, vol. 168, no. 3, pp. 765-771, 1993.

[9] C. Chalmers, "Does hysterectomy in a premenopausal woman affect ovarian function?" Medical Hypotheses, vol. 46, no. 6, pp. 573-575, 1996.

[10] A. Z. Souza, A. M. Fonseca, and V. M. Izzo, "Ovarian histology and function after total abdominal hysterectomy," Obstetrics and Gynecology, vol. 68, no. 6, pp. 847-849, 1986.

[11] T. Vuorento, J. Maenpaa, and I. Huhtaniemi, "Follow-up of ovarian endocrine function in premenopausal women after hysterectomy by daily measurements of salivary progesterone," Clinical Endocrinology, vol. 36, no. 5, pp. 505-510, 1992.

[12] J. G. Derksen, H. A. Brölmann, M. A. Wiegerinck, H. L. Vader, and A. P. M. Heintz, "The effect of hysterectomy and endometrial ablation on follicle stimulating hormone (FSH) levels up to 1 year after surgery," Maturitas, vol. 29, no. 2, pp. 133-138, 1998.

[13] R. Marana, M. Rizzi, L. Muzii, G. F. Catalano, P. Caruana, and S. Mancuso, "Correlation between the american fertility society classifications of adnexal adhesions and distal tubal occlusion, salpingoscopy, and reproductive outcome in tubal surgery," Fertility and Sterility, vol. 64, no. 5, pp. 924-929, 1995.

[14] B. Tiras, V. Noyan, H. Ozdemir, H. Guner, A. Yildiz, and M. Yildirim, "The changes in ovarian hormone levels and ovarian artery blood flow rate after laparoscopic tubal sterilization," European Journal of Obstetrics Gynecology and Reproductive Biology, vol. 99, no. 2, pp. 219-221, 2001.

[15] G. P. Gentile, S. C. Kaufman, and D. W. Heibig, "Is there any evidence for a post-tubal sterilization syndrome?" Fertility and Sterility, vol. 69, no. 2, pp. 179-186, 1998.

[16] A. S. Cevrioglu, B. Degirmenci, M. Acar et al., "Examination of changes caused by tubal sterilization in ovarian hormone secretion and uterine and ovarian artery blood flow rates," Contraception, vol. 70, no. 6, pp. 467-473, 2004.

[17] I. Thranov, J. B. Hertz, J. J. Kjer et al., "Hormonal and menstrual changes after laparoscopic sterilization by Falope- rings or Filshie-clips," Fertility and Sterility, vol. 57, no. 4, pp. 751-755, 1992.

[18] S. Kelekci, B. Yilmaz, Y. Yakut, L. Yasar, K. Savan, and S. Sonmez, "Hormonal and ovarian stromal blood supply changes after laparoscopic tubal sterilization: a prospective controlled study," Contraception, vol. 73, no. 3, pp. 279-283, 2006.

[19] J. A. Simpson, R. J. MacInnis, D. R. English, D. M. Gertig, H. A. Morris, and G. G. Giles, "A comparison of estradiol levels between women with a hysterectomy and ovarian conservation and women with an intact uterus," Climacteric, vol. 8, no. 3, pp. 300-303, 2005. 
[20] C. Chalmers, M. Lindsay, D. Usher, P. Warner, D. Evans, and M. Ferguson, "Hysterectomy and ovarian function: levels of follicle stimulating hormone and incidence of menopausal symptoms are not affected by hysterectomy in women under age 45 years," Climacteric, vol. 5, no. 4, pp. 366-373, 2002.

[21] S. Özdamar, H. Ülger, H. C. Sorkun, and I. Müderris, "Effects of hysterectomy on ovarian morphology and serum FSH level in rats," Maturitas, vol. 52, no. 1, pp. 60-64, 2005.

[22] O. L. Tapisiz, T. Gungor, H. Aytan et al., "Does hysterectomy affect ovarian function? Histopathologic evaluation and serum $\mathrm{FSH}$, inhibin $\mathrm{A}$, and inhibin B levels in an experimental rat model," European Journal of Obstetrics Gynecology and Reproductive Biology, vol. 140, no. 1, pp. 61-66, 2008.

[23] R. Fanchin, L. M. Schonäuer, C. Righini, J. Guibourdenche, R. Frydman, and J. Taieb, "Serum anti-Müllerian hormone is more strongly related to ovarian follicular status than serum inhibin B, estradiol, FSH and LH on day 3," Human Reproduction, vol. 18, no. 2, pp. 323-327, 2003.

[24] A. La Marca, G. Stabile, A. Carducci Artenisio, and A. Volpe, "Serum anti-Mullerian hormone throughout the human menstrual cycle," Human Reproduction, vol. 21, no. 12, pp. 3103-3107, 2006.

[25] C. Atabekoğlu, S. Taşkin, K. Kahraman et al., "The effects of total abdominal hysterectomy on serum anti-Müllerian hormone levels: a pilot study," Climacteric, vol. 15, no. 4, pp. 393-397, 2012. 


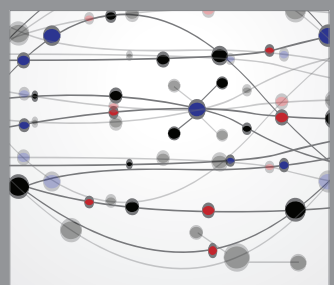

The Scientific World Journal
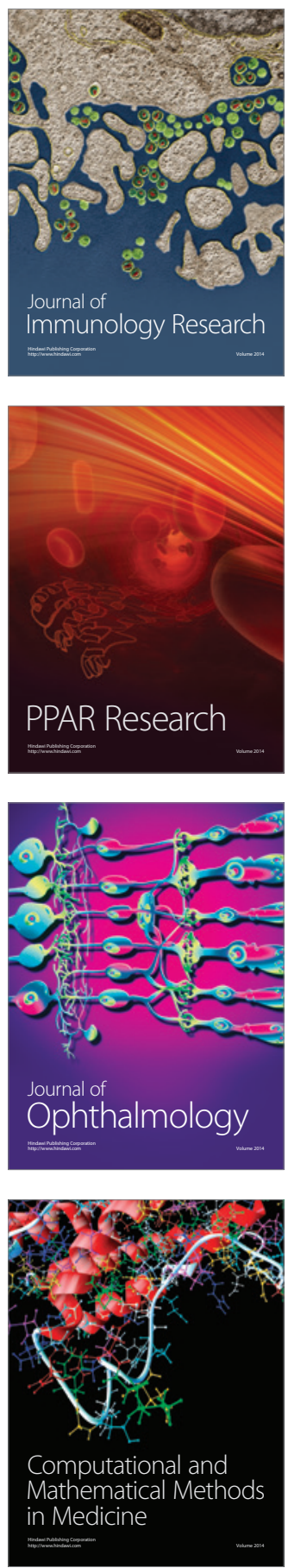

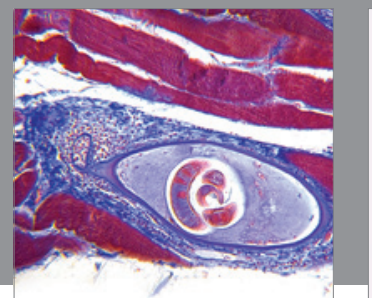

Gastroenterology

Research and Practice
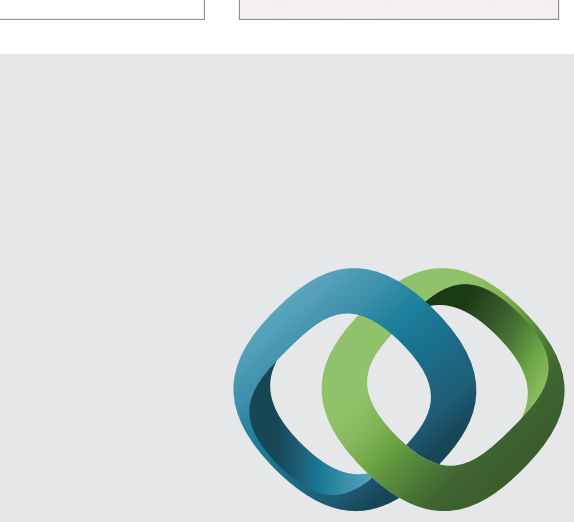

\section{Hindawi}

Submit your manuscripts at

http://www.hindawi.com
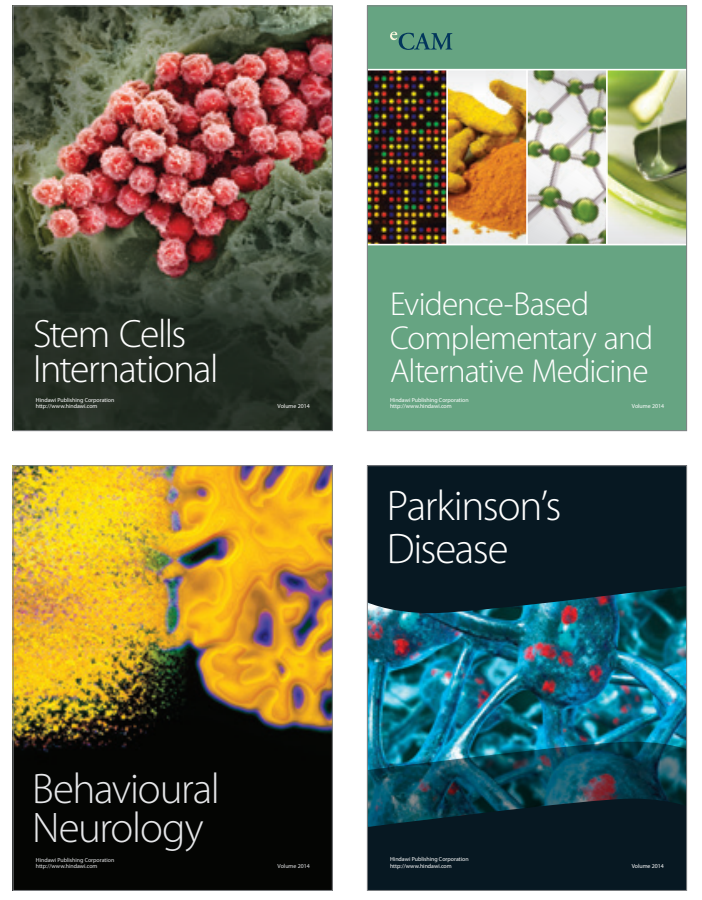
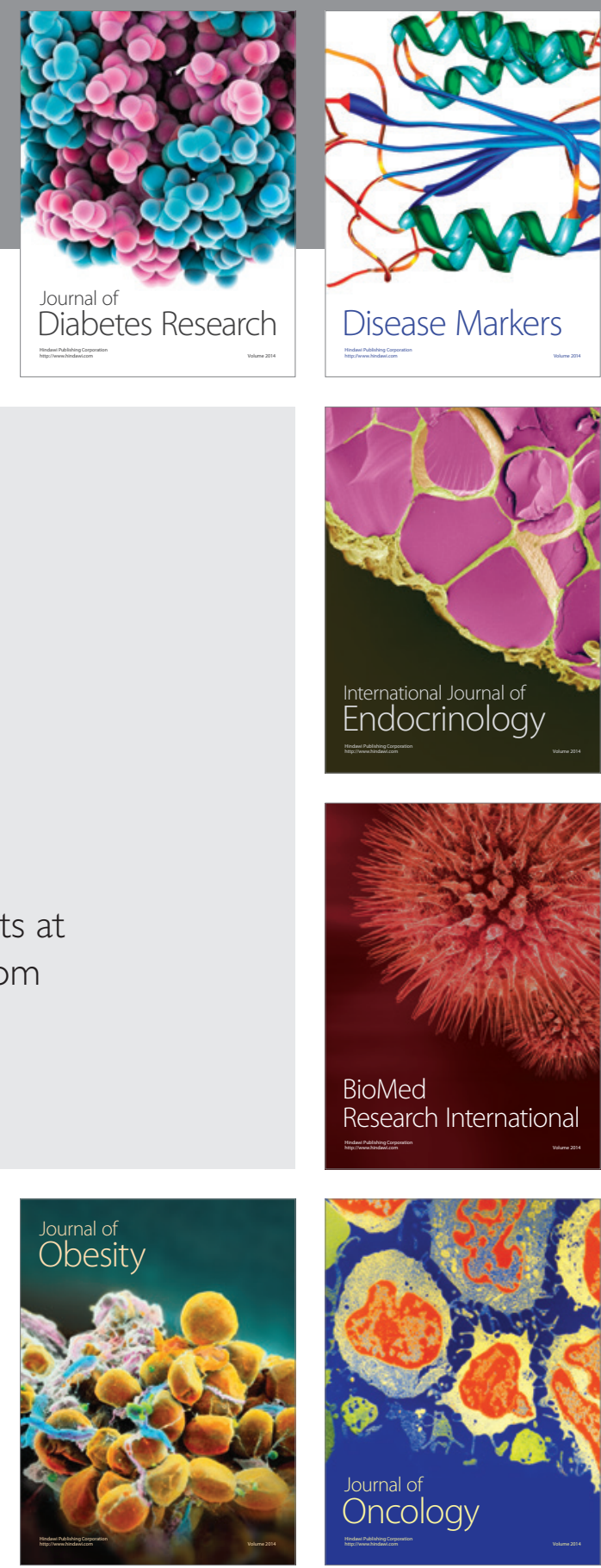

Disease Markers
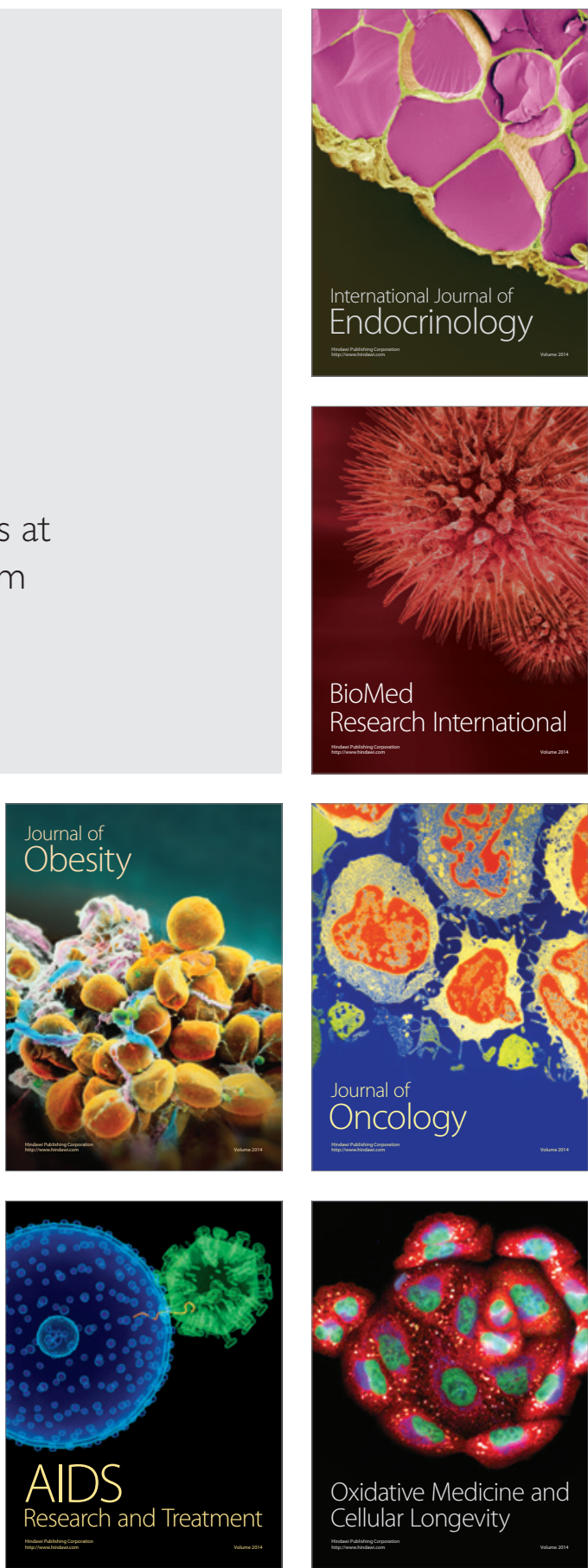